\title{
Efectos del método de gestión de residuos de arbolado afectado por incendio forestal en la regeneración del pinar en el sureste peninsular
}

\author{
Gómez-Sánchez, E. . ${ }^{*}$, Aroca, A. ${ }^{2}$, Marín, A. ${ }^{2}$ Lucas-Borja, M.E ${ }^{3}$ \\ ${ }^{1}$ Servicio de Montes y Espacios Naturales Protegidos. Servicios Periféricos de la Consejería de Agricultura en \\ Albacete. Junta de Comunidades de Castilla la Mancha. C/Mayor 47 02071. Albacete (España). \\ ${ }^{2}$ Empresa pública TRAGSA. C/Alonso Vidal nº 9, 02001. Albacete (España) \\ ${ }^{3}$ Universidad de Castilla la Mancha. Campus Universitario s/n, 02071. Albacete (España) \\ *e-mail: megomez@jccm.es
}

\section{Resumen}

El 1 julio de 2012 se inició en el sureste de la provincia de Albacete un incendio forestal que afectó a 5450 ha de su territorio. Para analizar el efecto de tres métodos de gestión de residuos forestales quemados (el acordonado, la trituración y la no actuación o dispersión de restos) en la regeneración de la especie arbórea principal (Pinus halepensis), se seleccionaron tres zonas arboladas afectadas representativas, realizando tres transectos por cada una de ellas: uno a lo largo de línea de restos acordonados $(\mathrm{Cr})$, otro sobre línea de restos triturados (Tr) y otro sin actuación (Ntr-Ncr), situando sobre cada uno cinco parcelas de muestreo de $1 \mathrm{~m}^{2}$. Se programaron dos muestreos, uno en mayo de 2013 coincidiendo con el fin de los trabajos de restauración, y el segundo en octubre de 2014. Los resultados muestran que unos tratamientos son más beneficiosos que otros en términos de supervivencia de plántulas de pino carrasco, siendo el más conveniente el no tratamiento de residuos o la no actuación, seguido de la trituración, resultando más desfavorable el acordonado de restos. En lo que respecta al crecimiento de los brinzales ninguno supone un aumento significativo, alcanzándose una menor altura media en la trituración.

Palabras clave: Masa mixta, nicho de regeneración óptimo, modelo de celda, regresión logística 


\section{Introducción}

El aumento de grandes incendios forestales en la cuenca mediterránea en las últimas décadas (Pausas y Fernández-Muñoz, 2012), donde los efectos ecológicos del fuego se han superpuesto a otras perturbaciones históricas y al cambio de las condiciones climáticas, ha puesto de manifiesto la necesidad de la restauración ecológico forestal de los ecosistemas quemados a fin de devolverles en el menor plazo posible su estructura, funcionalidad y resiliencia a las perturbaciones (Holling, 1963; Vallejo, 2009). Todo ello en el marco global de la lucha contra la desertificación.

La realización de cualquier actuación restauradora post-incendio debe ser planificada atendiendo a las características de la zona, la severidad del fuego y los objetivos de restauración establecidos; con la inevitable consideración del coste económico y la evaluación de los efectos positivos o negativos de cada una de las actuaciones a realizar. Dada la gran variabilidad de situaciones a las que el gestor de una zona quemada debe enfrentarse se hace necesario la experimentación con alternativas de manejo variadas que permitan una adecuada toma de decisiones en cada caso (Castro, et al., 2009)

Por otra parte, las zonas mediterráneas semiáridas están particularmente afectadas por los procesos de degradación de suelos, especialmente sobre litologías desfavorables como son los sustratos margosos (López-Bermúdez y Albaladejo, 1990). En general estas zonas se caracterizan por una cubierta vegetal escasa sometida a estrés hídrico, suelos muy pobres en materia orgánica con un alto riesgo potencial de erosión; de manera que cuando un incendio forestal recorre estas superficies la recuperación de estos ecosistemas se convierte en un proceso lento y difícil, que fácilmente puede verse alterado por una nueva perturbación (Bautista, 1999). La ejecución de actuaciones urgentes encaminadas a evitar el riesgo de degradación y la pérdida de suelos deben ser las primeras a desarrollar en estas zonas incendiadas.

Los mulches o cubiertas orgánicas están cada vez más extendidos como técnicas de rehabilitación forestal, empleándose materiales de distinta naturaleza como la paja, cebada o trigo; astillas o virutas de madera y corteza, e incluso compost (Aguilar, 1999; Napper, 2006; Gimeno et al., 2009) siempre con el objetivo de proteger el suelo del impacto de la lluvia, disminuir la velocidad de la escorrentía superficial y favorecer el mantenimiento de la humedad en el suelo (Gimeno et al., 2009). Muchos han sido los estudios que han verificado los efectos beneficiosos sobre el suelo de los mulches (Bautista, 1999; Robichaud et al., 2013; Vega, 2014); si bien pocos son los estudios relativos a sus efectos en la regeneración de la vegetación, habiéndose obtenido resultados distintos (Fernández y Vega, 2014; Santana et al., 2014).

Por otro lado, y dentro de la intensa polémica en torno a la conveniencia o no de realizar la extracción de la madera quemada, el abandono de los restos de madera quemada in situ se posiciona como uno de los argumentos en contra de dicha extracción al suponer una fuente importante de nutrientes cuya incorporación gradual al suelo repercute en la actividad de organismos descomponedores, en las tasas de reciclaje y en definitiva en la disponibilidad de nutrientes para las plantas (Castro et al., 2013).

El objetivo de este trabajo es el de analizar el mejor método de tratamiento de re- 
siduos forestales generados después de un incendio desde el punto de vista de la regeneración natural de la especie arbórea principal. Se pretende evaluar qué tratamiento post-incendio de los residuos forestales (acordonado, la trituración y la no actuación (dispersión de restos) favorece una mayor tasa de supervivencia y crecimiento del regenerado de pino carrasco (Pinus halepensis) en una zona incendiada del sureste de España. Como hipótesis de partida se considera que el acordonado, dado su efecto reductor de la insolación, puede mejorar la tasa de supervivencia en comparación con el triturado o no actuación (abandono de restos dispersos); y que el triturado reduce la supervivencia del regenerado preexistente al dañarlo por efecto mecánico.

\section{Material y métodos}

\subsection{Descripción zona del incendio}

El estudio se ha llevado a cabo en la cuenca de Prado Piñero, sobre terrenos de monte público afectados por el incendio de Sierra Seca y Donceles de Hellín de 1 de julio de 2012 que afectó a 5470 ha de la provincia de Albacete (Fig. 1). La altitud de la cuenca de 495 ha oscila entre 330 m del Río Segura y los 790 m de su cima más

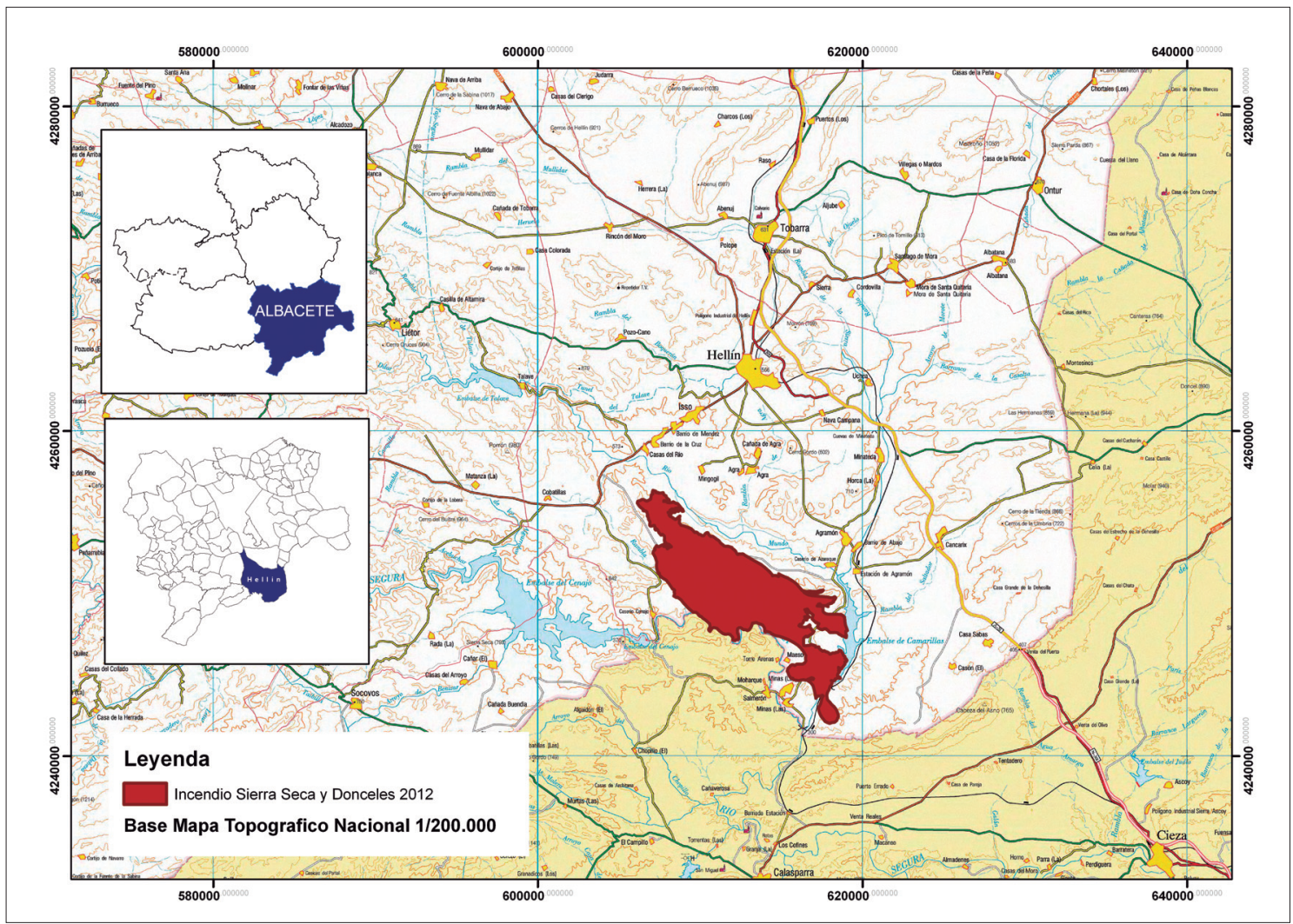

Figura 1. Mapa de localización del incendio de Sierra Seca y Donceles de 1/07/2012 
alta; encontrándonos en el piso bioclimático mesomediterráneo (temperatura media de $17^{\circ} \mathrm{C}$, máxima de $40^{\circ} \mathrm{C}$ ) con un ombroclima semiárido (precipitación media de 320 $\mathrm{mm}$ ). Los materiales que conforman la cuenca son poco resistentes (margas, arenas y coluviones) a excepción de los crestones calizos. En sus laderas antes del incendio se desarrollaba una vegetación compuesta por pino carrasco (Pinus halepensis), procedente de repoblaciones realizadas hace 30 años por la Administración Forestal mediante subsolado y aterrazado, de muy escaso desarrollo; con un sotobosque de Stipa tenacissima y otras especies térmicas de escasa cobertura (Anthyllis cytisoides, Cistus albidus, Teucrium pseudochamaepitys, Lapiedra martinezii).

\subsection{Parcelas y tratamientos}

Los trabajos de restauración post-incendio realizados en la cuenca de Prado Piñero estuvieron destinados a disminuir el riesgo de erosión hídrica en la época otoñal. Con este fin se apearon y procesaron los pies quemados empleando sus fustes para la construcción de albarradas en las cárcavas de menos de un metro de profundidad y en taludes de grandes barrancos. El material sobrante (restos) fue acordonado siguiendo curvas de nivel ante la carencia de material en cantidad y tamaño suficiente para su empleo en la construcción de fajinas; en una pequeña superficie estos residuos fueron triturados con desbrozadora de cadenas.

Con objeto de analizar qué método de gestión de residuos producía efectos más beneficiosos en el establecimiento y crecimiento de la especie arbórea principal (Pinus halepensis) se seleccionaron tres zonas representativas de la variabilidad de las laderas bajas y medias de la cuenca (Fig. 2). Las zona A y B se encuentran separadas cerca de $250 \mathrm{~m}$, ambas con una altitud de $410 \mathrm{~m}$ aproximadamente presentando diferencias en cuanto a la litología y orientación; la zona A presenta orientación Noroeste con suelo desarrollado sobre areniscas y calizas y la zona B presenta orientación sureste y litología margosa. La zona $\mathrm{C}$, situada a $800 \mathrm{~m}$ al noroeste de las anteriores, tiene una altitud de 460 metros con exposición a todos los vientos y suelo de naturaleza margosa, con mayor presencia de afloramientos de costras calizas por su mayor proximidad a las zonas de pendiente.

Cada tratamiento se caracterizó por su ratio de cobertura, sombreado y precio según tarifas de la Empresa Pública TRAGSA para el año 2013 (Tab. 1); destacando la escasa cobertura del tratamiento de trituración consecuencia del escaso volumen y tamaño de restos.

Tabla 1. Ratio de cobertura, sombreado y precio (Tarifas TRAGSA 2011 actualizadas a 2013 en http://www.tragsa.es) para cada tratamiento.

\begin{tabular}{cccc}
\hline Tratamiento & Cobertura & Sombreado & Precio (€/ha) \\
\hline Sin actuación & $0 \%$ & NO & 0 \\
\hline Acordonado & $60 \%$ & SI & 457.58 \\
\hline Triturado & $5 \%-10 \%$ & NO & 670.10 \\
\hline
\end{tabular}




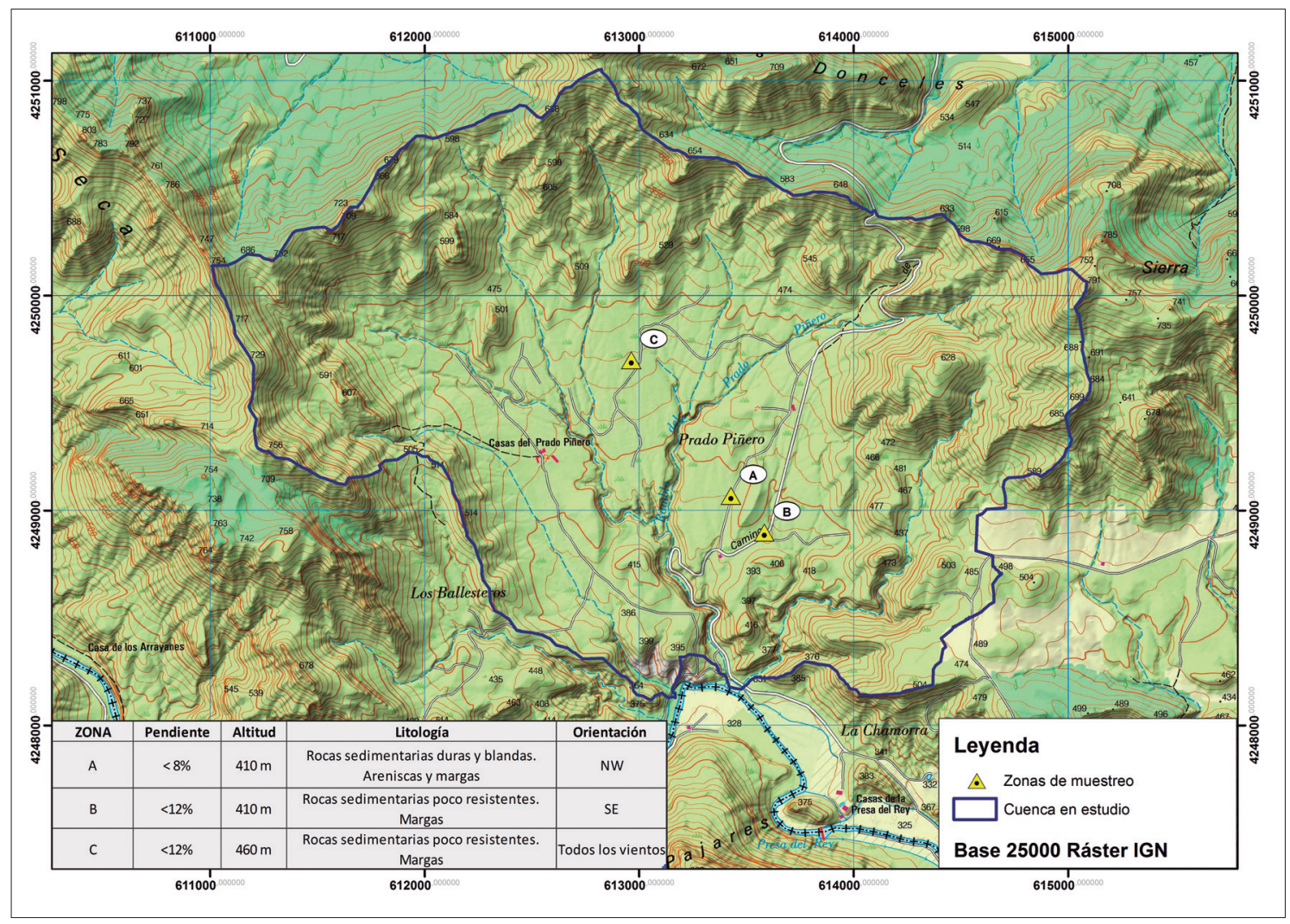

Figura 2. Mapa de localización de las zonas de tratamiento en la cuenca en estudio.

En cada zona se señalaron tres transectos de 25 metros de longitud: uno a lo largo de línea de restos acordonados (Cr), otro sobre línea de restos triturados (Tr) y otro con restos dispersos sin actuación (Ncr_Ntr). En cada transecto se situaron cinco parcelas de muestreo de $1 \mathrm{~m} 2$ distanciadas 4 metros entre ellas. Durante la selección de las zonas de estudio se puso cuidado para escoger superficies no afectadas por los trabajos previos de construcción de fajinas y albarradas.

En el presente trabajo se han realizado dos muestreos. El primer muestro se realizó en mayo de 2013, una vez finalizados todos los trabajos de restauración, contabilizándose el regenerado de pino carrasco en cada subparcela y anotando los individuos dañados por la trituración (rotura del ápice por la cadena de la desbrozadora). De esta manera, se contabilizó la regeneración existente antes de aplicar ningún tratamiento de eliminación de restos, sumando la regeneración después de los tratamientos y las plántulas dañadas o muertas. El segundo muestreo se realizó en octubre de 2014, contabilizándose las plántulas vivas y muertas. En ambos casos se midió la altura de los brinzales vivos.

A fin de detectar diferencias significativas en la regeneración, supervivencia y crecimiento de pino carrasco según zonas y tratamientos se realizaron sucesivos test ANOVA unifactoriales. Entre zonas se estudia la relación entre el regenerado inicial (plántulas ha-1) y porcentaje de supervivencia (\%) a fin de detectar diferencias significativas que puedan ser explicadas por condiciones microambientales, que cada tra- 
tamiento post-incendio aplicado genera. Seguidamente se estudia el efecto de la trituración sobre el regenerado calculando la densidad de plántulas dañadas por unidad de superficie estimando el porcentaje medio de plántulas dañadas considerando todas las subparcelas tratadas (45 réplicas), dado que en todas ellas la trituración se realizó con el mismo sistema (desbrozadora de cadenas). Finalmente, se realiza el test estadístico entre el incremento en la altura de plántula entre muestreos (crecimiento) y el porcentaje de supervivencia (\%) observado en muestreo de 2014 con respecto a muestreo de 2013 según la zona y el método de gestión de residuos realizado.

\section{Resultados}

\subsection{Primer muestreo}

La regeneración postincendio de pino carrasco es irregular entre zonas existiendo diferencias significativas $(\mathrm{F}=10.60$, g.l. $=2, \mathrm{p}=0.0107$, siendo $\mathrm{F}$ el estadístico que refleja el grado de similitud de las medias comparadas, g.l. los grados de libertad y p el nivel de significación). La zona con más regenerado inicial es la zona $\mathrm{B}(9.53 \pm 2.27$ plántulas $\left.\mathrm{m}^{-2}\right)$, seguido de la zona A $\left(4.53 \pm 2.72\right.$ plántulas $\left.\mathrm{m}^{-2}\right)$ y la zona C $(1.47 \pm 1.22$ plántulas $\left.\mathrm{m}^{-2}\right)$; sin que existan diferencias significativas en la altura de las plántulas vivas entre zonas $(\mathrm{F}=0.422$, g.1. $=2, \mathrm{p}=0.674)$, con una media de $6.07 \pm 1.10 \mathrm{~cm}$.

En cuanto al porcentaje de marras obtenido en el primer muestreo (antes de la realización de los tratamientos), no se observan diferencias significativas entre zonas $(\mathrm{F}=1.164$ g.l. $=2, \mathrm{p}=0.733)$ con una media de $8.86 \pm 15.26 \%$ (Fig. 3). El triturado de los residuos daña el $17.45 \pm 21.36 \%$ de las plántulas, ocasionando la rotura de la parte superior de la plántula y su posterior desaparición.

\subsection{Segundo muestreo}

En este muestreo el regenerado ha disminuido considerablemente en comparación con el primero, existiendo diferencias significativas entre zonas $(\mathrm{F}=10.28$, g.l. $=2$, $\mathrm{p}=0.0115$ ), si bien responde a la misma tendencia inicial (Fig.4). La zona con más regenerado es la zona $\mathrm{B}\left(3.22 \pm 0.87\right.$ plántulas $\left.\mathrm{m}^{-2}\right)$, seguido de la zona $\mathrm{A}(0.86 \pm 1.17$ plántulas $\left.\mathrm{m}^{-2}\right)$ y la zona $\mathrm{C}\left(0.20 \pm 0.20\right.$ plántulas $\left.\mathrm{m}^{-2}\right)$; sin que exista diferencias significativas en la altura de los brinzales vivos entre zonas $(\mathrm{F}=0.422$, g.l. $=2, \mathrm{p}=0.674)$, con una media de $12.52 \pm 1.98 \mathrm{~cm}$.

Las principales causas del alto porcentaje de marras observado se justifican en las escasas precipitaciones tras la nascencia de las plántulas, destacando las registradas en 2014 en comparación con la media histórica (Tab. 2), y la predación soportada por los brinzales ante la falta de otros recursos alimenticios disponibles a los herbívoros.

En cuanto al porcentaje de mortandad por zonas, y sin diferenciar tratamientos, el test estadístico indica que no hay diferencias significativas $(\mathrm{F}=0.343, \mathrm{~g} .1 .=2$, $\mathrm{p}=0.722$ ). 


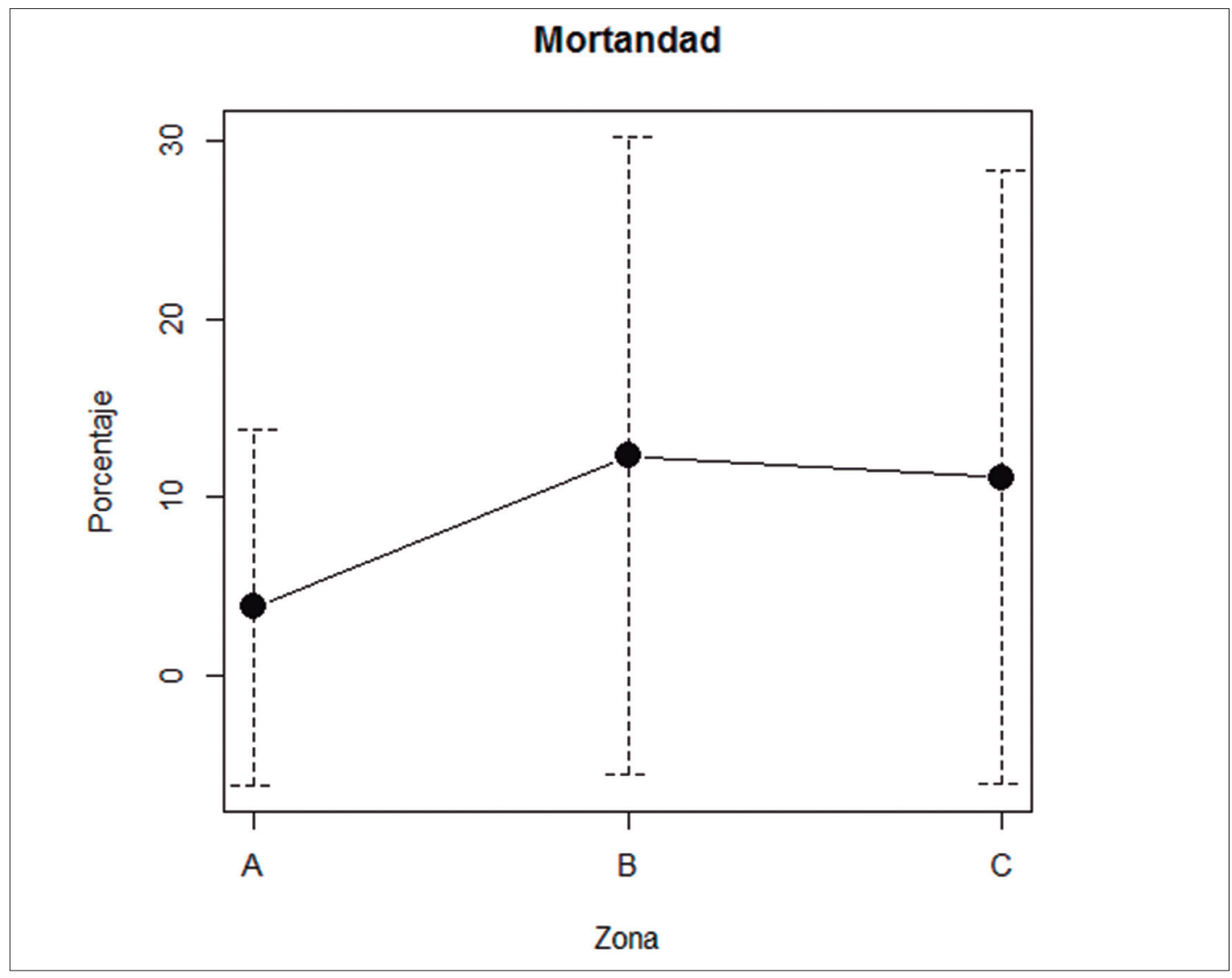

Figura 3. Densidad de regenerado inicial $\left(\right.$ plántulas $\mathrm{m}^{-2}$ ) y porcentaje de mortandad de plántulas de pino carrasco en el muestreo 1.

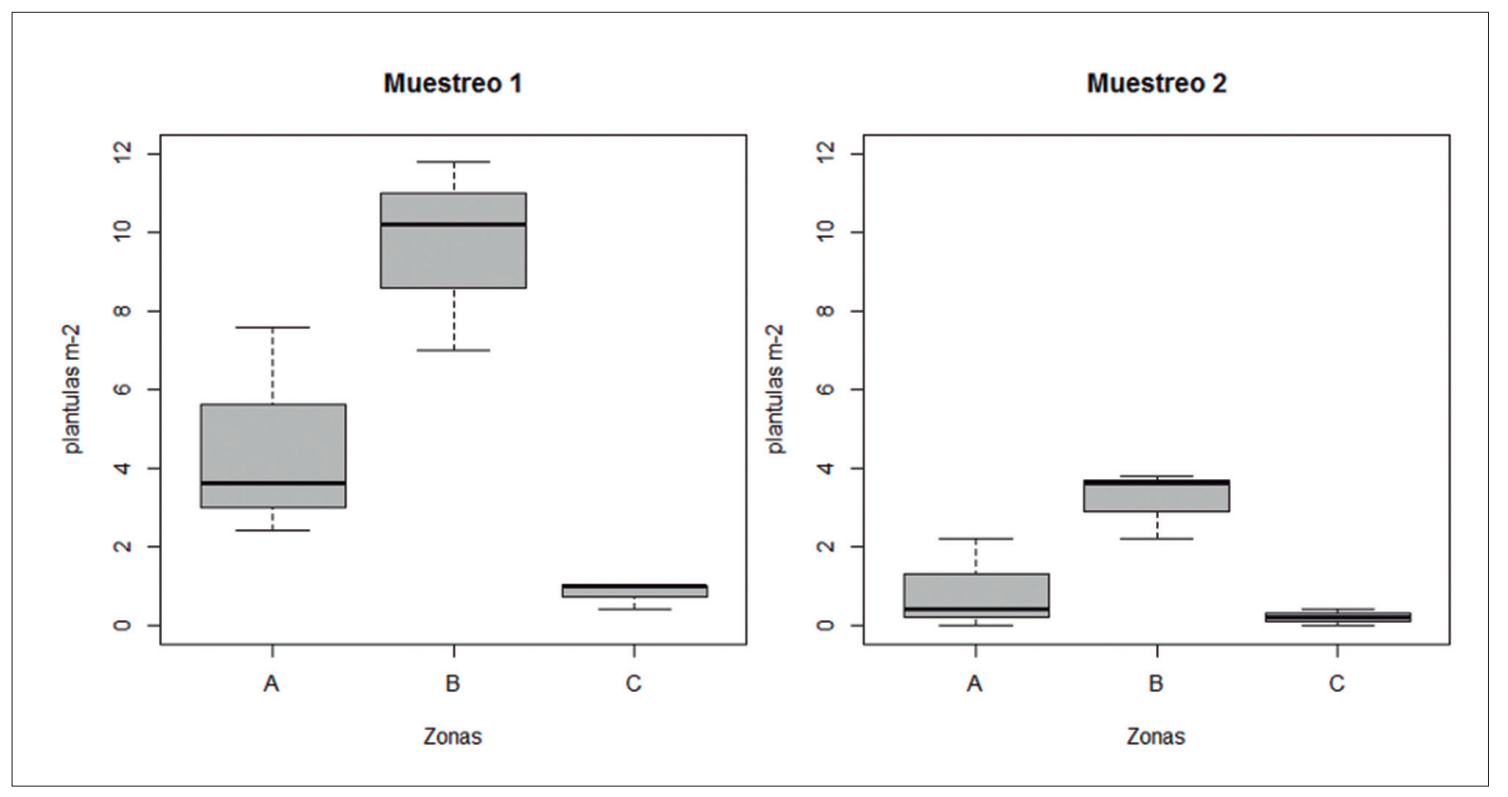

Figura 4. Densidad de plántulas vivas (plántulas $\mathrm{m}^{-2}$ ) en muestreo 1 y 2. 
Tabla 2. Distribución mensual de precipitaciones $(\mathrm{mm})$ desde incendio hasta segundo muestreo comparado con el promedio histórico (periodo 1945-2013).

Año 2012

\begin{tabular}{|c|c|c|c|c|c|c|c|c|c|c|c|c|c|}
\hline Mes & & & & & & & jul & ago & sep & oct & nov & dic & Total \\
\hline $\mathrm{P}(\mathrm{mm})$ & & & & & & & 0 & 16.50 & 65.20 & 53.60 & 92.00 & 0.10 & 227.40 \\
\hline \multicolumn{14}{|c|}{ Año 2013} \\
\hline Mes & ene & feb & mar & abr & may & jun & jul & ago & sep & oct & nov & dic & Total \\
\hline $\mathrm{P}(\mathrm{mm})$ & 5.50 & 25.70 & 38.60 & 52.60 & 19.00 & 0.10 & 1.00 & 57.20 & 42.00 & 25.00 & 19.40 & 23.50 & 309.60 \\
\hline \multicolumn{14}{|c|}{ Año 2014} \\
\hline Mes & ene & feb & mar & $a b r$ & may & jun & jul & ago & sep & oct & & & Total \\
\hline $\mathrm{P}(\mathrm{mm})$ & 6.30 & 7.80 & 6.67 & 19.00 & 10.20 & 13.10 & 1.50 & 0 & 9.50 & 9.30 & & & 83.37 \\
\hline \multicolumn{14}{|c|}{ Media (1945-2013) } \\
\hline Mes & ene & feb & mar & abr & may & jun & jul & ago & sep & oct & nov & dic & Total \\
\hline $\mathrm{P}(\mathrm{mm})$ & 20.11 & 22.25 & 28.15 & 38.12 & 39.61 & 20.93 & 8.02 & 15.31 & 32.97 & 44.59 & 28.98 & 22.57 & 321.55 \\
\hline
\end{tabular}

Sí se detectan diferencias significativas entre el porcentaje de supervivencia de plántulas entre los distintos métodos de eliminación de residuos $(\mathrm{F}=7.794$, g.1. $=2$, $\mathrm{p}=0.0215)$. El mayor porcentaje lo encontramos en los transectos no tratados $(46.07 \pm 3.44 \%)$ seguido de los triturados $(38.89 \pm 5.09 \%) \mathrm{y}$, llamativamente por debajo, los acordonados $(20.46 \pm 3.43 \%$ ) (Fig. 5).

En términos de crecimiento de plántulas, expresado en incremento de altura entre muestreos, no existen diferencias significativas entre tratamientos $(\mathrm{F}=1.918$, g.l. $=2$, $\mathrm{p}=0.227$ ). Igual entre alturas totales alcanzadas, sin bien la media es algo inferior en el tratamiento trituración (Fig. 6).

\section{Discusión}

La regeneración post-incendio de pino carrasco en la cuenca en estudio es relativamente baja comparada con otras zonas próximas quemadas, observándose una distribución irregular dentro de la cuenca debido a cambios en las condiciones microambientales que condicionan tanto la densidad y el tamaño del regenerado. Este cambio ejerce una mayor influencia en climas áridos donde el pino carrasco se encuentra sometido a fuerte estrés hídrico. Hemos de señalar que estas condiciones parecen condicionar más la producción de semillas de los pies adultos (función del número y tamaño de piñas), y por tanto la densidad inicial de regenerado, que el éxito en el establecimiento inicial de las plántulas emergidas.

En lo que respecta a la comparación de los resultados de supervivencia entre tratamientos es llamativo que sea el tratamiento de acordonado de residuos el que presenta menor porcentaje de supervivencia de plántulas y que éste no haya llevado aparejado un mejor desarrollo de la planta. La hipótesis planteada de partida fue que, dadas las altas temperaturas y escasas precipitaciones en la zona, la disminu- 


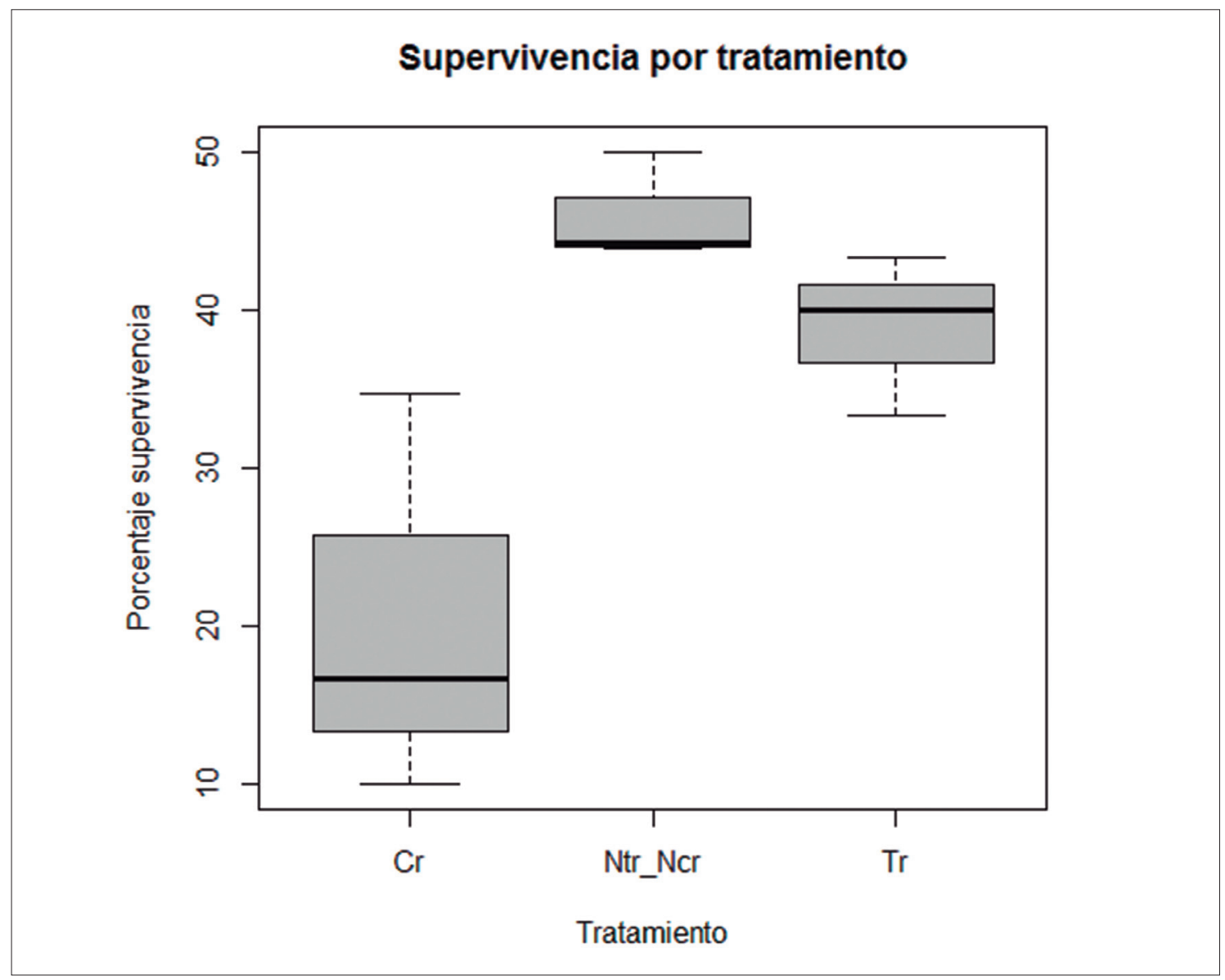

Figura 5. Porcentaje de supervivencia de plántulas de pino carrasco por tratamiento en muestreo 2

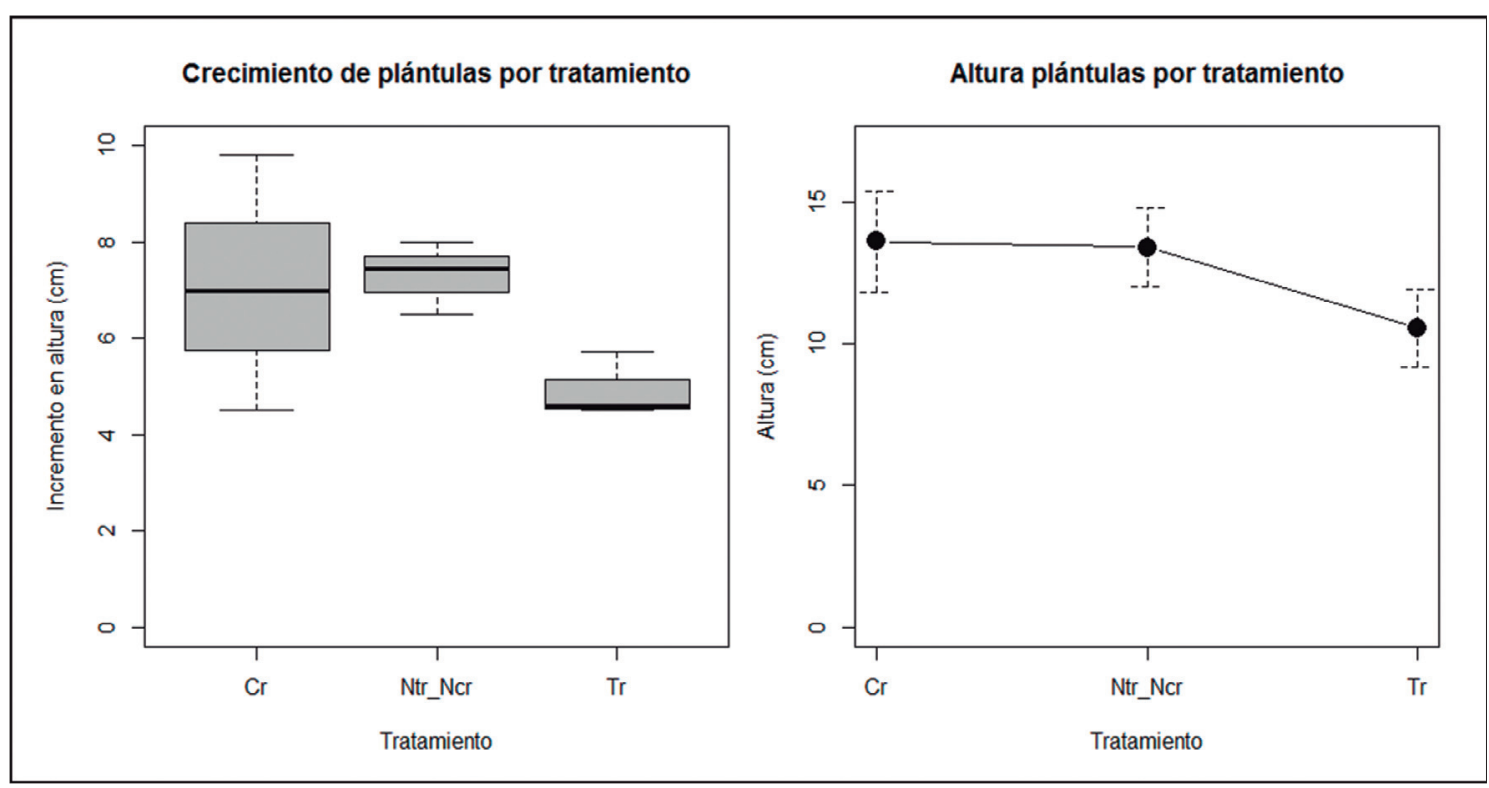

Figura 6. Crecimiento en altura registrado entre muestreos y altura de plántula alcanzada según tratamiento en muestreo 2 
ción de la temperatura en los primeros centímetros del suelo junto con el aumento del porcentaje de humedad ocasionado por la cubierta (Santana et al., 2014) mejoraría tanto la supervivencia como el desarrollo de las plántulas. En este caso, no sólo la cubierta no tiene el efecto neutro o ligeramente positivo en el establecimiento de las plántulas observada en estudio similares (Dodson and Peterson, 2010; Fernández y Vega, 2013) sino que tiene un efecto negativo en la supervivencia de pino carrasco, en clara contraposición con las conclusiones de otros estudios (Santana et al., 2014). Ello podría estar relacionado con la falta de luz requerida en los primeros estadios de desarrollo por las especies de temperamento robusto y/o la barrera física que pueden ejercer los restos acordonados sobre las plántulas (Dodson and Peterson, 2010).

Comparado el porcentaje de supervivencia de plántulas entre la trituración y la no actuación, y considerando el porcentaje de mortandad ocasionado por la desbrozado$\mathrm{ra}$, se desaconseja la eliminación de residuos mediante triturado en la zona argumentando: (a) el nulo efecto en la supervivencia de plántulas, (b) la menor altura que estas alcanzan con este tratamiento y que puede deberse a la rotura de las plántulas por la cadena de la desbrozadora, (c) la escasa cobertura que ejercen los restos triturados sobre el suelo que nos impiden considerar beneficios en lo que a la reducción de la erosión hídrica y (d) el coste económico. No obstante, en esta afirmación se puede estar obviando la mejora que supone la mayor velocidad en la descomposición de los restos triturados e incorporación al suelo en una zona donde la erosión hídrica ocasiona importes pérdidas y cuyos efectos pueden ser a más largo plazo.

Los resultados obtenidos para el tratamiento de trituración no nos deben hacer pensar que la aplicación de un mulch de astillas con una cobertura adecuada no pudiera tener efectos positivos en la zona, ya que en estos casos obviaríamos la mortandad de plántulas producida en la trituración y sumaríamos los efectos positivos sobre la erosión hídrica y la incorporación de nutrientes. Igualmente no se deben extender los resultados a otras zonas donde la variación de las condiciones climáticas y de vegetación pueden convertir el astillado en una alternativa viable ecológica y económicamente.

Por último, cabe indicar que la metodología a seguir en cualquier trabajo de experimentación es fundamental, siendo especialmente importante en el caso de trabajos de investigación a largo plazo, como es el caso que nos ocupa. En este sentido, sería aconsejable prolongar los muestreos en el tiempo y modificar el tamaño de las parcelas, con el objetivo de tener datos más fiables. Según el protocolo para la obtención de datos en trabajos de regeneración natural redactado por Bravo y Rodríguez-García (2005), se recomienda una malla cuadrada de $100 \mathrm{~m}$ de lado y orientado según la orografía del terreno.

\section{Conclusiones}

Una vez analizados los datos y concluidos los trabajos de restauración en esta etapa preliminar se puede afirmar en base a los resultados obtenidos que: 
- El mejor método de tratamiento de los residuos en la zona de estudio ha sido el "no tratamiento" o abandono de los restos dispersos por el monte en términos de supervivencia y crecimiento de regenerado de pino carrasco.

- El acordonado de restos disminuye la supervivencia de plántulas de pino carrasco sin inhibir su crecimiento.

- Se desaconseja la trituración de restos, salvo en aquellos casos en que la densidad de astilla obtenida sea tal que produzca efectos positivos que compensen los nulos y/o negativos demostrados con este tratamiento.

- Para confirmar los resultados y conclusiones aquí expuestas, se recomienda seguir el protocolo para la obtención de datos en trabajos de regeneración natural redactado por Bravo y Rodríguez-García (2005).

\section{Referencias}

Albaladejo, J., Díaz, E., 1990. Degradación y regeneración del suelo en el litoral mediterráneo español: experiencias en el proyecto LUCDEME. Degradación y regeneración del suelo en condiciones ambientales mediterráneas,191-214.

Alloza, J.A., García-Barreda, S., Gimeno, T., Vallejo, R., Rojo, L., Martínez, A., 2013. Guía técnica para la gestión de montes quemados. Protocolos de actuación para la restauración de zonas quemadas con riesgo de desertificación. Madrid. España.

Bautista, S., 1999. Regeneración post-incendio de un pinar (Pinus halepensis Mill) en ambiente semiárido. Erosión de suelo y medidas de conservación a corto plazo. Tesis doctoral. Universidad de Alicante.

Bravo, F., Rodríguez-García, E., 2005. Protocolo para la obtención de datos. Regeneración natural y primer desarrollo de rodales forestales en el centro de España. http://www.inia. es/GCONTREC/PUB/PROTOCOLO_REGENERACION.UVA_1138894450734.pdf . Acceso. 05 Enero 2015

Dodson, E.K., Peterson, D.W., 2010. Mulching effects on vegetation recovery following high severity wildfire in north-central Washington State, USA. For. Ecol. Manag. 260(10), 1816-1823.

Fernández, C., Vega, J.A., 2014. Efficacy of bark strands and straw mulching after wildfire in NW Spain: Effects on erosion control and vegetation recovery. Ecol. Eng. 63, 50-57.

Gimeno, T., Bladé, C., Haroun., K., Susana, B., 2006. Tratamientos de rehabilitación post-incendio en áreas sensibles a la degradación del suelo. IV Congreso Forestal Español.

Holling, C.S., 1963. Resilience and stability of ecological Systems. Annu. Rev. Ecol. Syst. $4(1), 1-23$

Napper, C., 2006. BAER: Burned Area Emergency Response Treatments Catalog. San Dimas, California.

Pausas, J.G., Fernández-Muñoz, S., 2012. Fire regime changes in the Western Mediterranean Basin: from fuel-limited to drought-driven fire regime. Climatic Change 110(1-2), 215-226.

Ramón Vallejo, V., Bautista Aguilar, S., Alloza Millán, J., 2009. Restauración de montes quemados en condiciones mediterráneas. Recursos Rurais, IBADER: Instituto de Biodiversidade Agraria e Desenvolvemento Rural. 5, 55-60. 
Robichaud, P.R., Jordán, P., Lewis, S.A., Ashmun, L.E., Covert, S.A., Brown, R.E., 2013. Evaluating the effectiveness of wood shred and agricultural straw mulches as a treatment to reduce post-wildfire hillslope erosion in southern British Columbia, Canada. Geomorphology 197, 21-33.

Santana, V.M., Alday, J.G., Baeza, M.J., 2014. Mulch application as post-fire rehabilitation treatment does not affect vegetation recovery in ecosystems dominated by obligate seeders. Ecol. Eng. 71, 80-86.

Vega, J. A., Fernández, C., Fonturbel, T., González-Prieto, S., Jiménez, E., 2014. Testing the effects of straw mulching and herb seeding on soil erosion after fire in a gorse shrubland. Geoderma 223, 79-87. 